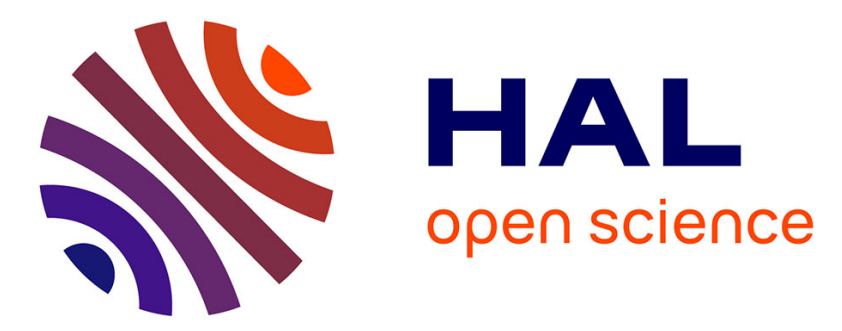

\title{
Activité reproductrice de la pyrale du maïs, Ostrinia nubilalis Hbn. (Lep. Pyralidae) à basses températures constantes
}

Hossain Gohari, Nicole Hawlitzky

\section{- To cite this version:}

Hossain Gohari, Nicole Hawlitzky. Activité reproductrice de la pyrale du maïs, Ostrinia nubilalis Hbn. (Lep. Pyralidae) à basses températures constantes. Agronomie, 1986, 6 (10), pp.911-917. hal-00884839

\section{HAL Id: hal-00884839 \\ https://hal.science/hal-00884839}

Submitted on 1 Jan 1986

HAL is a multi-disciplinary open access archive for the deposit and dissemination of scientific research documents, whether they are published or not. The documents may come from teaching and research institutions in France or abroad, or from public or private research centers.
L'archive ouverte pluridisciplinaire HAL, est destinée au dépôt et à la diffusion de documents scientifiques de niveau recherche, publiés ou non, émanant des établissements d'enseignement et de recherche français ou étrangers, des laboratoires publics ou privés. 


\title{
Activité reproductrice de la pyrale du maïs, Ostrinia nubilalis Hbn. (Lep. Pyralidae) à bas- ses températures constantes
}

\author{
Hossain GOHARI \& Nicole HAWLITZKY \\ I.N.R.A., Station de Zoologie, Centre de Recherches de Versailles, Route de St-Cyr, F 78000 Versailles.
}

\begin{abstract}
Différentes expressions de l'activité reproductrice d'Ostrinia nubilalis Hbn., telles que l'accouplement et les aspects quantitatifs et qualitatifs de la ponte, ont été étudiées dans une gamme de températures constantes relativement basses comprises entre 11 et $16^{\circ} \mathrm{C}$. L'expérimentation a été menée dans les conditions de laboratoire, avec une photopériode de 16 heures sur des pyrales dont les chenilles ont été collectées dans la nature au cours des printemps 1982 et 1983 . L'accouplement a lieu rarement au-dessous de $13{ }^{\circ} \mathrm{C}$. L'activité de ponte devient négligeable en dessous d'un seuil thermique dont la valeur a varié selon l'année de récolte des chenilles dans la nature $\left(15^{\circ} \mathrm{C}\right.$ en 1982 et $13{ }^{\circ} \mathrm{C}$ en 1983). En dessous de ces valeurs, se produisent une diminution du nombre des femelles pondeuses, celles du nombre de pontes et d'œufs déposés par femelle et une augmentation du nombre d'œut's stériles. Le nombre d'œufs par ooplaque ne varie pas en fonction de la température. Dans la gamme des températures relativement basses où l'activité de ponte se produit à un niveau encore assez élevé, la baisse de température a un effet progressif, et l'émission des œufs s'étend sur une période plus longue qu'à $25{ }^{\circ} \mathrm{C}$, la température de référence.
\end{abstract}

Mots clés additionnels : Fécondité, fertilité, accouplement, longévité.

\begin{abstract}
Reproductive activity of the European corn borer, Ostrinia nubilalis Hbn. (Lep. Pyralidae) under constant low temperatures.

Various aspects of the reproductive activity of the European corn borer, Ostrinia nubilalis, have been studied at relatively constant low temperatures ranging from 11 to $16^{\circ} \mathrm{C}$. The experiment was run under laboratory conditions with a 16-hour light period on adults whose larvae had been collected in nature during the spring of 1982 and 1983 . Mating rarely occurred below $13{ }^{\circ} \mathrm{C}$. Oviposition became negligible below a temperature threshold whose value changed during the two years of the experiment $\left(15^{\circ} \mathrm{C}\right.$ in 1982 and $13{ }^{\circ} \mathrm{C}$ in 1983). Below these values the number of fecund females and of eggs per female decreased considerably and many deposited eggs were sterile. The mean number of eggs per egg-mass did not change with temperature. Over the relatively low temperature range where oviposition occured at a rather high level, decline in temperature had a progressive effect, and oviposition lasted for a longer period during the adult life than at $25^{\circ} \mathrm{C}$ (reference temperature).
\end{abstract}

Additional key words : Fecundity, fertility, mating, longevity.

\section{INTRODUCTION}

Le contrôle des insectes ravageurs par lâchers d'insectes oophages nécessite de connaître d'une part le début du vol et de la ponte, d'autre part l'évolution et l'intensité de cette dernière durant la totalité de la période où elle a lieu chez le ravageur.

Chez la pyrale du maïs, les seuils thermiques inférieurs de développement des différents stades ont été étudiés (CAFFREY \& WORTHLEY, 1927 ; GUENNELON \& AUdEMARD, 1960 ; MATTESON \& DECKER, 1965) et ont permis le calcul de sommes de degrés jours qui sont à la base de méthodes de prévision du début de vol, et de la ponte (APPLE, 1952; JACKSON \& PETERS, 1963 ; JARVIS \& BRINDLEY, 1965 ; LOEWER et al., 1974 ; CLÉMENT et al., 1981 ; STENGEL, 1982 ; HAWLITZKY, 1986). Par contre, il existe peu de travaux sur les seuils thermiques inférieurs d'accouplement et d'oviposition chez la pyrale. BARBER (1925) et STIRRETT (1938) ont remarqué une diminution de l'intensité de ponte en fonction d'une baisse de température dans la nature. VANCE (1949) a démontré cet 
effet dans des conditions de températures constantes ou alternées au laboratoire sur une race américaine sans chercher à définir avec précision le seuil thermique inférieur.

La connaissance de ces seuils permettrait d'apprécier les conséquences immédiates de baisses de température hors saison qui peuvent se produire durant la période d'activité reproductrice des populations naturelles et de mieux comprendre le comportement de ponte traduit par l'intensité de celle-ci, en fonction des variations thermiques. L'objectif de ce travail a donc été de faire une approche de ces seuils dans des conditions constantes de température pour des populations de pyrale du nord de la France.

\section{MATÉRIEL ET MÉTHODES}

Les adultes sur lesquels porte cette étude, effectuée pendant 2 années consécutives, sont issus de chenilles dont le lieu d'origine diffère selon l'année. Les chenilles récoltées en juin 1982 sont originaires de Beauce (Levesville, 28) et celles récoltées en juin 1983, originaires de la région de Houdan (28).

Ces chenilles au $5^{\mathrm{e}}$ stade, et dont la diapause était achevée, ont été extraites de tiges de maïs stockées en cage depuis l'automne précédent. Les larves sont mises à $25^{\circ} \mathrm{C}$ dans des boîtes en polystyrène transparent $(26 \times 13 \times 7,5 \mathrm{~cm})$ contenant des fragments de tiges, jusqu'à leur transformation en chrysalides. Les chrysalides sont maintenues à cette température et placées isolément dans des piluliers contenant du sable humide et fermés par un bouchon perforé. Les émergences des papillons sont contrôlées chaque matin et les adultes sont transférés immédiatement dans une étuve réglée à la température à laquelle l'activité de ponte est étudiée. Les papillons sont mis par couple dans des cages cylindriques de $8 \mathrm{~cm}$ de diamètre et $10 \mathrm{~cm}$ de hauteur, dont la partie inférieure repose sur du sable humide. La face supérieure grillagée est recouverte d'un papier filtre destiné à recevoir les pontes, sur lequel est posée une éponge synthétique humidifiée régulièrement. L'alimentation hydrique est assurée par un morceau de coton gorgé d'eau placé dans une petite soucoupe. Les papiers filtres sont changés journellement le matin (rappelons que la pyrale du maîs pond la nuit), les cages étant sorties pendant un temps très court dans la chambre climatisée à $5{ }^{\circ} \mathrm{C}$ où se trouvent les étuves renfermant les couples ; ceci, afin de ne pas provoquer une éventuelle stimulation de l'activité reproductrice par exposition à une température supérieure à celles testées. Le choix de ces dernières a été effectué en se basant sur l'unique température, signalée dans la littérature comme responsable d'un arrêt total de la ponte. (VANCE, 1949). Les températures ainsi retenues se situent soit à son niveau soit en dessous, afin de cerner le phénomène avec un maximum de précision. Du fait de cette recherche de précision, il a été nécessaire d'avoir une gamme de températures décroissantes de $1^{\circ}$ en $1{ }^{\circ} \mathrm{C}$. Ceci a restreint le nombre de couples mis en expérience, d'une part par manque d'enceintes climatisées, d'autre part en raison de la multiplication des comptages à réaliser au niveau des caractères observés, la rigueur de l'expérimentation nécessitant que l'on tra- vaille simultanément sur l'ensemble des séries. Ainsi l'accouplement et l'activité de ponte de 15 couples de pyrales sont étudiés à 4 températures constantes la première année $\left(13,14,15\right.$ et $\left.16^{\circ} \mathrm{C}\right)$ et en fonction des résultats obtenus, à 5 températures $\left(11\right.$ à $\left.15^{\circ} \mathrm{C}\right)$ la seconde année. Lors des 2 années d'expérimentation, 15 couples témoins sont placés à $25^{\circ} \mathrm{C}$ car cette température est proche de l'optimum thermique pour la ponte (GAHUKAR, 1976). Chaque température varie de $\pm 0,5^{\circ} \mathrm{C}$ et l'humidité relative oscille entre 75 et 90 p. 100 , du début à la fin de l'expérimentation. La photopériode est de $16 \mathrm{~h}$ dans les étuves et dans la salle d'élevage à $25^{\circ} \mathrm{C}$.

Les caractères observés sont les suivants :

- longévité,

- présence ou absence de spermatophores dans la bourse copulatrice (dissection des femelles après leur mort),

- nombre d'ooplaques déposées par chaque femelle et nombre d'œufs les constituant (fécondité journalière et totale),

- fertilité des œufs,

- nombre de femelles pondeuses.

\section{RÉSULTATS}

\section{A. Longévité}

Sur l'ensemble des 2 années, on peut considérer que les femelles ont tendance à vivre plus longtemps que les mâles et qu'en dessous de $16{ }^{\circ} \mathrm{C}$ la longévité est 2 à 4 fois plus grande qu'à $25^{\circ} \mathrm{C}$, tout en étant variable d'une année à l'autre (tabl. 1).

\section{TABLEAU 1}

Longévité moyenne (en jours) de la pyrale à différentes températures. Les chiffres entre parenthèses représentent les écarts types.

Mean longevity in days of Ostrinia nubilalis at different temperatures. The figures in brackets are the standard deviations.

\begin{tabular}{|c|c|c|c|c|}
\hline \multirow[b]{2}{*}{$\mathrm{t}^{\circ} \mathrm{C}$} & \multicolumn{2}{|c|}{ été 82} & \multicolumn{2}{|c|}{ été 83} \\
\hline & $0^{*}$ & q & $\sigma$ & Q \\
\hline 11 & & & $\begin{array}{c}29,3 \\
(12,9)\end{array}$ & $\begin{array}{r}38,2 \\
(12,7)\end{array}$ \\
\hline 12 & & & $\begin{array}{c}42,6 \\
(15,8)\end{array}$ & $\begin{array}{c}34 \\
(13,6)\end{array}$ \\
\hline 13 & $\begin{array}{c}53,6 \\
(16,3)\end{array}$ & $\begin{array}{c}57,2 \\
(19,6)\end{array}$ & $\begin{array}{c}38,1 \\
(15,3)\end{array}$ & $\begin{array}{c}41,1 \\
(14,8)\end{array}$ \\
\hline 14 & $\begin{array}{l}24,1 \\
(9,4)\end{array}$ & $\begin{array}{c}47,1 \\
(10,5)\end{array}$ & $\begin{array}{c}41,5 \\
(12,7)\end{array}$ & $\begin{array}{r}47,1 \\
(12,5)\end{array}$ \\
\hline 15 & $\begin{array}{c}29,5 \\
(12,5)\end{array}$ & $\begin{array}{c}31,4 \\
(13,1)\end{array}$ & $\begin{array}{c}33 \\
(10,8)\end{array}$ & $\begin{array}{l}33,4 \\
(8,9)\end{array}$ \\
\hline 16 & $\begin{array}{c}26,8 \\
(11,7)\end{array}$ & $\begin{array}{c}30,7 \\
(9,9)\end{array}$ & & \\
\hline 25 & $\begin{array}{l}13 \\
(3,3)\end{array}$ & $\begin{array}{l}14,4 \\
(3,5)\end{array}$ & $\begin{array}{l}10,4 \\
(4,5)\end{array}$ & $\begin{array}{c}10,73 \\
\text { (3) }\end{array}$ \\
\hline
\end{tabular}




\section{B. Accouplement}

Rappelons que nous avons utilisé la présence de spermatophore comme indice d'accouplement. Chaque spermatophore présent dans la bourse copulatrice correspond à un accouplement durant la vie de la femelle (PESHO, 1961).

Chez les pyrales issues de chenilles de 1981-1982 et dans la limite des températures étudiées, l'accouplement semble avoir lieu aussi bien à la temperature minimale testée $\left(13^{\circ} \mathrm{C}\right)$ qu'à celle maximale $\left(25^{\circ} \mathrm{C}\right)$. Par contre, chez les pyrales issues de chenilles de 1982-1983 on constate, au-dessous de $13{ }^{\circ} \mathrm{C}$, une nette augmentation du nombre des femelles chez qui l'accouplement n'a pas eu lieu. Lors des 2 années d'expérimentation, au-dessus de $13{ }^{\circ} \mathrm{C}$ le cas le plus fréquent est la présence d'un seul spermatophore (tabl. 2). Une corrélation entre la fréquence des accouplements chez les femelles et leur longévité n'est pas mise en évidence, les coefficients de corrélation étant tous non-significatifs (tabl. 2).
TABLEAU 2

Pourcentages, à différentes températures, des femelles ayant eu $0(a), 1(b), 2$ (c) spermatophores ou plus $(d) . c c=$ coefficients de corrélation entre la longévité des femelles et la fréquence d'accouplement (d.d.I $=13, r=0,51$ au risque de $5 \%$ ).

Percentage of females at different temperatures with $0(a), 1$ (b), 2 (c) or more (d) spermatophores. $c c=$ correlation coefficients between female longevity and mating frequency (with degrees of freedom $=13 ; r=0.51$ at the $5 \%$ level).

\begin{tabular}{ccccccccccc}
\hline & \multicolumn{4}{c}{} & \multicolumn{3}{c}{ été 1982} & & \multicolumn{5}{c}{ été 1983} \\
\cline { 2 - 12 }$t{ }^{\circ} C$ & $a$ & $b$ & $c$ & $d$ & $c c$ & $a$ & $b$ & $c$ & $d$ & $c c$ \\
\hline 11 & & & & & & 73 & 27 & 0 & 0 & $-0,30$ \\
\hline 12 & & & & & & 73 & 20 & 7 & 0 & 0,49 \\
\hline 13 & 13 & 67 & 13 & 7 & $-0,11$ & 7 & 73 & 20 & 0 & 0,02 \\
\hline 14 & 64 & 29 & 7 & 0 & $-0,11$ & 20 & 73 & 7 & 0 & 0,23 \\
\hline 15 & 7 & 79 & 14 & 0 & 0,25 & 7 & 79 & 7 & 7 & 0,23 \\
\hline 16 & 33,3 & 53,3 & 13,3 & 0 & 0,37 & & & & \\
\hline 25 & 0 & 93 & 7 & 0 & 0,18 & 13 & 80 & 7 & 0 & 0,35 \\
\hline \hline
\end{tabular}

TABLEAU 3

Paramètres ayant trait à la fécondité. Les chiffres entre parenthèses représentent les écarts-types. Parameters concerned with the fecundity of females. The figures in brackets are standard deviations.

a. 1982

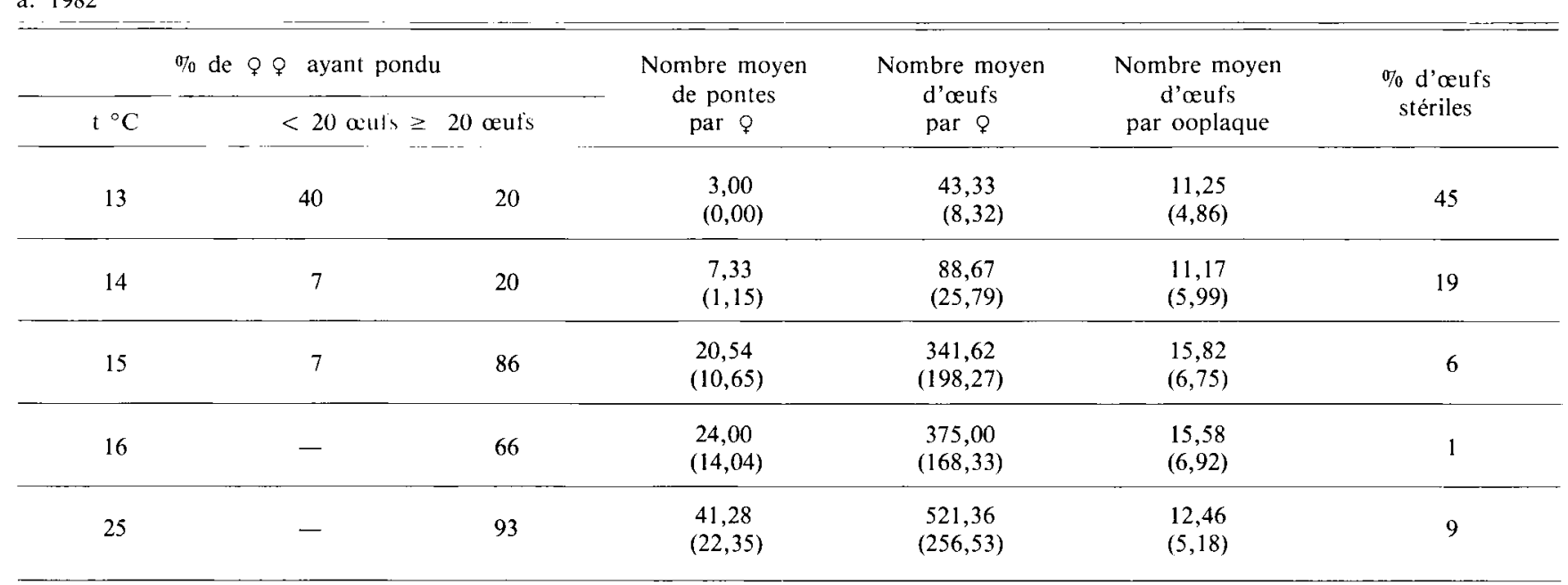

b. 1983

\begin{tabular}{|c|c|c|c|c|c|c|}
\hline \multicolumn{3}{|c|}{$\%$ de $q Q$ ayant pondu } & \multirow{2}{*}{$\begin{array}{c}\text { Nombre moyen } \\
\text { de pontes } \\
\text { par } ९\end{array}$} & \multirow{2}{*}{$\begin{array}{c}\text { Nombre moyen } \\
\text { d'œufs } \\
\text { par } 9\end{array}$} & \multirow{2}{*}{$\begin{array}{c}\text { Nombre moyen } \\
\text { d'œufs } \\
\text { par ooplaque }\end{array}$} & \multirow{2}{*}{$\begin{array}{l}\% \text { d'œufs } \\
\text { stériles }\end{array}$} \\
\hline $\mathrm{t}^{\circ} \mathrm{C}$ & $<20$ outs $\geq$ & 20 outs & & & & \\
\hline 11 & 7 & 13 & $\begin{array}{c}6,50 \\
(2,12)\end{array}$ & $\begin{array}{c}91,00 \\
(36,77)\end{array}$ & $\begin{array}{l}13,42 \\
(5,28)\end{array}$ & 38 \\
\hline 12 & 14 & 13 & $\begin{array}{c}7,00 \\
(1,41)\end{array}$ & $\begin{array}{l}89,00 \\
(8,48)\end{array}$ & $\begin{array}{l}11,40 \\
(5,38)\end{array}$ & 62 \\
\hline 13 & 7 & 53 & $\begin{array}{l}20,00 \\
(7,07)\end{array}$ & $\begin{array}{l}259,50 \\
(99,67)\end{array}$ & $\begin{array}{l}12,09 \\
(5,17)\end{array}$ & 24 \\
\hline 14 & 14 & 73 & $\begin{array}{c}15,11 \\
(16,53)\end{array}$ & $\begin{array}{c}165,73 \\
(186,80)\end{array}$ & $\begin{array}{l}11,87 \\
(5,44)\end{array}$ & 21 \\
\hline 15 & - & 93 & $\begin{array}{l}27,21 \\
(12,18)\end{array}$ & $\begin{array}{c}396,57 \\
(175,52)\end{array}$ & $\begin{array}{l}14,21 \\
(4,94)\end{array}$ & 6 \\
\hline 25 & 7 & 66 & $\begin{array}{c}39,70 \\
(15,23)\end{array}$ & $\begin{array}{c}537,20 \\
(161,08)\end{array}$ & $\begin{array}{l}13,35 \\
(4,68)\end{array}$ & 0 \\
\hline
\end{tabular}




\section{Fécondité totale et fertilité des oufs}

Différentes expressions de la fécondité sont prises en considération pour étudier l'effet de températures basses sur la fécondité totale :

- le pourcentage de femelles pondeuses à l'intérieur de chaque série,

- le nombre d'ooplaques et d'œufs déposés par femelle pondeuse,

- le nombre moyen d'œufs par ooplaque.

Dans le tableau 3 figure le pourcentage de femelles qui ont pondu plus ou moins de $20 œ u$ fs au total durant toute leur vie. Les femelles ayant pondu moins de 20 œufs ne sont pas prises en compte dans le calcul des moyennes afin de réduire la dispersion des résultats à l'intérieur de chaque série expérimentale.

On remarque qu'une baisse importante du pourcentage des femelles pondeuses et de la fécondité (nombre d'œufs ou d'ooplaques par femelle pondeuse) se produit entre 15 et $14{ }^{\circ} \mathrm{C}$ chez les femelles de 1981-1982 (tabl. 3a) et entre 13 et $12{ }^{\circ} \mathrm{C}$ chez celles de 1982-1983 (tabl. 3b). Les coefficients de corrélation calculés entre la quantité d'œufs déposés et la longévité des femelles d'une part, entre cette quantité et le nombre d'accouplements d'autre part, ne sont pas significatifs au risque de 5. p. 100 (tabl. 4).

Des œufs stériles sont observés aussi bien chez des femelles accouplées que chez celles non accouplées : la proportion des œufs stériles par rapport à la totalité des œufs est plus élevée aux températures auxquelles l'activité de ponte est réduite $\left(13^{\circ}\right.$ et $14^{\circ} \mathrm{C}$ en 1982 ; tabl. 3a) $\left(11^{\circ}\right.$ et $12^{\circ} \mathrm{C}$ en 1983 ; tabl. 3b).

\section{Rythme et étalement dans le temps de l'activité de ponte}

En étudiant l'activité journalière de ponte à chaque température, et femelle par femelle, on constate qu'à $25^{\circ} \mathrm{C}$, elle est continue jusqu'à son arrêt définitif. Aux températures basses où l'activité est réduite par rapport à celle observée à $25^{\circ} \mathrm{C}$, mais non négligeable ( 15 et $16^{\circ}$ en 1982 et 15 à $13^{\circ} \mathrm{C}$ en 1983 ), il peut $y$
TABLEAU 4

Coefficients de corrélation entre le nombre d'œufs pondus par les femelles et leur longévité ou le nombre d'accouplements. Les chiffres entre parenthèses représentent l'effectif des femelles ayant pondu. Aucun de ces coefficients n'est significatif au risque de $5 \%$. Correlation coefficients between the number of eggs deposited and the longevity or mating frequency of the females. Figures in brackets are the number of females having laid. None of these coefficients were significant at the $5 \%$ level.

\begin{tabular}{|c|c|c|c|c|}
\hline \multirow[b]{2}{*}{$\mathrm{t}^{\circ} \mathrm{C}$} & \multicolumn{2}{|c|}{1982} & \multicolumn{2}{|c|}{1983} \\
\hline & longévité & accouplement & longévité & accouplement \\
\hline \multicolumn{5}{|l|}{11} \\
\hline \multicolumn{5}{|l|}{12} \\
\hline 13 & & & $0,18 \quad(8)$ & $-0,26$ \\
\hline 14 & & & $-0,16(11)$ & $0,49(11)$ \\
\hline 15 & $0,29(13)$ & $0,35(13)$ & $0,11(14)$ & $-0,05(14)$ \\
\hline 16 & $0,22(10)$ & $0,22(10)$ & & \\
\hline 25 & $-0,19(14)$ & $0,05(14)$ & $0,60(10)$ & $0,10(10)$ \\
\hline
\end{tabular}

avoir une discontinuité dans le rythme de ponte due à l'existence de plusieurs jours pendant lesquels cesse l'activité de ponte. Chez les femelles qui ont pondu à 13 et $14^{\circ} \mathrm{C}$ en 1982 et à 11 et $12^{\circ} \mathrm{C}$ en 1983 l'activité de ponte, quantitativement négligeable, est complètement anarchique dans son rythme par rapport à celle observée à des températures plus élevées. La figure 1 montre pour chaque année l'activité de ponte au cours du temps, de 2 femelles choisies comme étant représentatives (profil de ponte le plus fréquemment observé), dans les séries situées en dessus et audessous de la température critique $\left(15\right.$ et $14{ }^{\circ} \mathrm{C}$ en 1982 et 13 et $12^{\circ} \mathrm{C}$ en 1983 ).

Nous avons représenté dans la figure 2 pour chaque série expérimentale le pourcentage cumulé du nombre d'œufs pondus en fonction de l'âge des femelles par rapport au nombre total d'œufs déposés. Avec une baisse de température l'activité de ponte s'étale sur

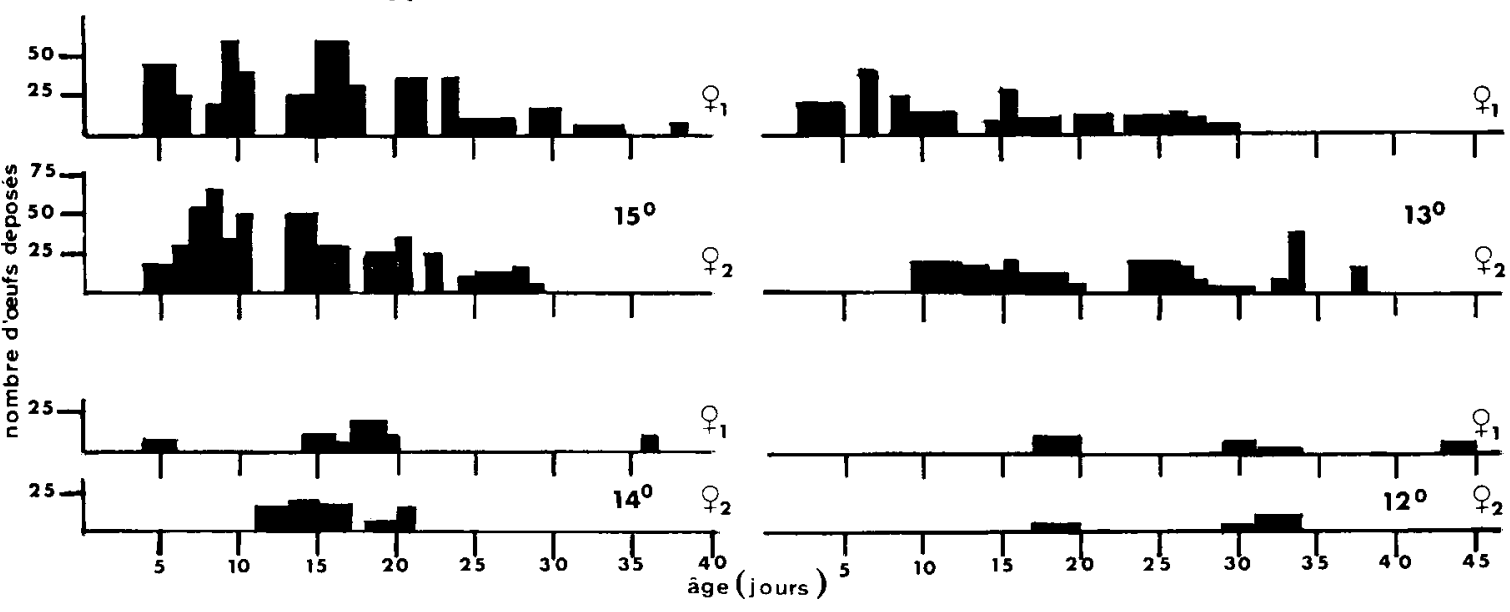

Figure 1 


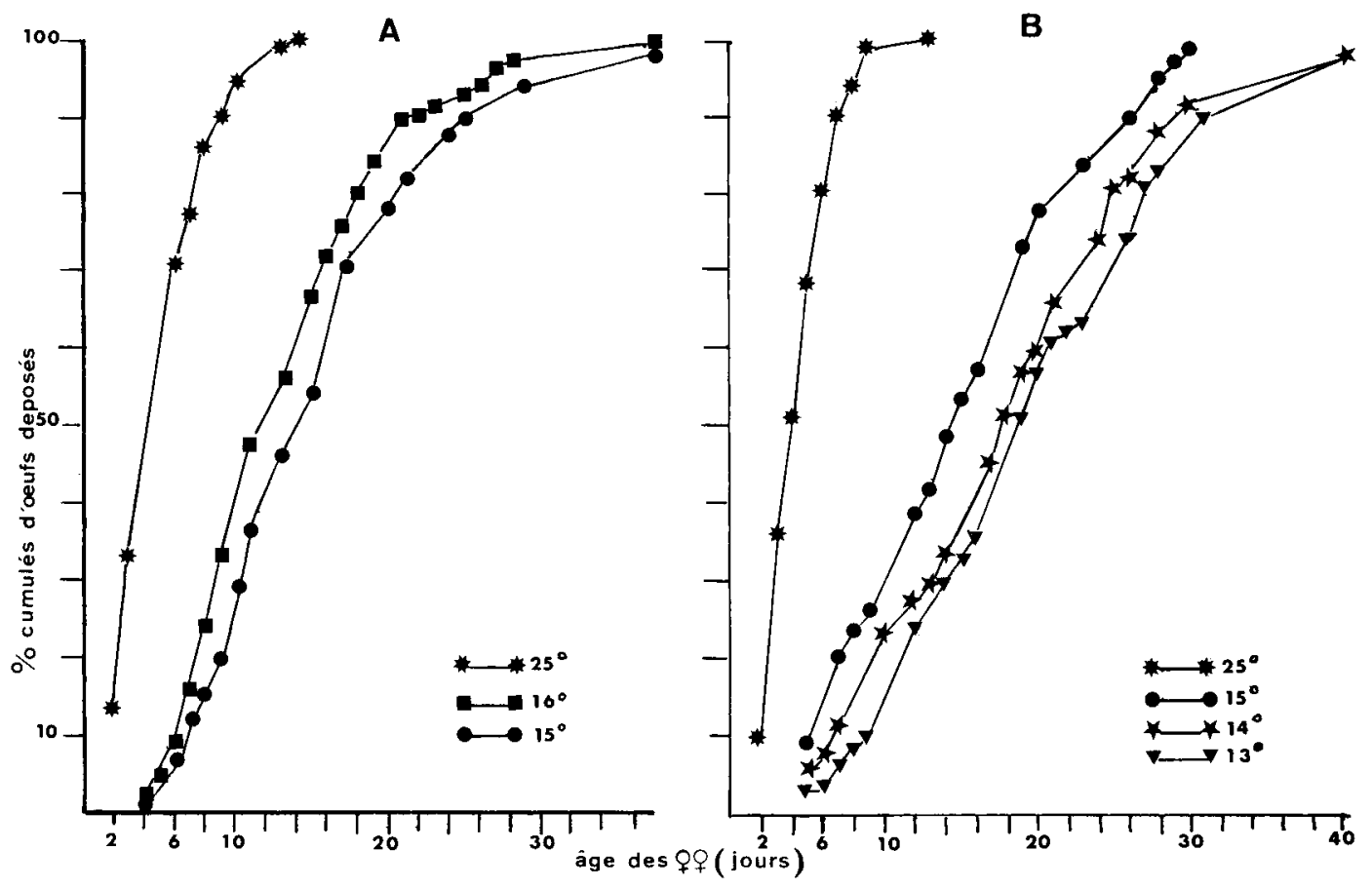

Figure 2

Pourcentage cumulé du nombre d'œufs pondus quotidiennement rapporté à la totalité d'œufs déposés par les fernelles en 1982 (A), en $1983(B)$

une période plus longue. Pour les pyrales de 19811982, 70 p. 100 des œufs sont déposés après 6, 15 et $17 \mathrm{j}$ respectivement à $25^{\circ}, 16^{\circ}$ et $15^{\circ} \mathrm{C}$. La même tendance s'observe pour les pyrales de 1982-1983 et ce même pourcentage est atteint après $5,17,22$ et $24 \mathrm{j}$ respectivement à $25^{\circ}, 15^{\circ}, 14^{\circ}$ et $13{ }^{\circ} \mathrm{C}$.

\section{E. Nombre moyen d'oufs par ooplaque}

Le nombre moyen d'œufs par ooplaque ne varie pas en fonction de la température. En effet, il est du même ordre de grandeur à 11 et $25^{\circ} \mathrm{C}$ (tabl. 3b). Etudié également en fonction de l'âge des femelles, ce paramètre est illustré par la figure $3 a, b$ où sont indiqués les coefficients de corrélation. Une des caractéristiques de ces résultats est la grande variation, pour un âge donné, du nombre moyen journalier d'œufs par ponte, d'une femelle à l'autre, au sein d'une même série. Il est visible également que cette variation est moins grande vers la fin de la vie et qu'en général elle l'est autant à $25^{\circ} \mathrm{C}$ qu'aux faibles températures. L'observation de ces figures et la valeur des coefficients de corrélation révèlent qu'il existe une liaison négative entre la taille de l'ooplaque et l'âge de la femelle. Mais c'est surtout vers la fin de la vie qu'il y a moins d'œufs par ponte déposée.

\section{DISCUSSION ET CONCLUSION}

Nous avons constaté qu'à température basse telle que $13{ }^{\circ} \mathrm{C}$, l'accouplement peut avoir lieu aussi bien qu'à température plus élevée comme $25^{\circ} \mathrm{C}$. Un effet nettement défavorable s'observe à 12 et $11^{\circ} \mathrm{C}$ où les accouplements, bien qu'existants, deviennent plus
Cumulative percentage of daily oviposition, relative to total eggs laid by all females in $1982(A)$, in 1983 (B).

rares. Cet effet peut être lié à une inactivité générale et en particulier à une impossibilité de voler à des températures aussi basses. STIRRETT (1938) \& BROERSMA et al. (1976) rapportent en effet que la température minimale pour le vol se situe entre 12 et $13{ }^{\circ} \mathrm{C}$.

Sur l'ensemble des 2 années se dégage une tendance à la stérilité des œufs aux températures testées inférieures à $15^{\circ} \mathrm{C}$. VANCE (1949) indique qu'en moyenne il y a beaucoup plus d'œufs stériles quand la température varie au cours de la vie qu'en température constante. La stérilité observée dans notre étude ne peut être attribuée à l'absence d'accouplement car on l'observe chez des femelles qui portent des spermatophores. On peut penser que les températures basses ont alors un effet défavorable sur la fécondation.

BARBER (1925), en s'appuyant sur ses observations dans la nature, constate qu'une température moyenne nocturne inférieure ou égale à $15,4^{\circ} \mathrm{C}\left(60^{\circ} \mathrm{F}\right.$ dans le texte) réduit significativement le nombre d'œufs déposés pendant la nuit correspondante. STIRRETT (1938), en analysant les résultats de 10 années d'observations dans les champs, constate qu'il n'y a pas de relation nette entre la température moyenne de chaque nuit et le nombre d'œufs déposés. VANCE (1949), en étudiant l'activité de ponte de la pyrale dans les conditions du laboratoire et à températures constantes, observe que la femelle cesse de pondre dès qu'elle est exposée à des températures inférieures ou égales à $15,4{ }^{\circ} \mathrm{C}$, mais cette température ne peut être considérée comme le seuil réel car VANCE n'a pas testé les températures intermédiaires entre $20,9{ }^{\circ} \mathrm{C}\left(70^{\circ} \mathrm{F}\right.$ dans le texte) et $15,4^{\circ} \mathrm{C}\left(60^{\circ} \mathrm{F}\right)$. Si on compare nos résultats à ceux de cet auteur, on constate que l'activité de ponte de la pyrale du nord de la France se maintient à une température bien inférieure $\left(11^{\circ} \mathrm{C}\right)$ à celle à laquelle la pyrale américaine cesse de pondre $\left(15^{\circ} \mathrm{C}\right)$. Par rap- 

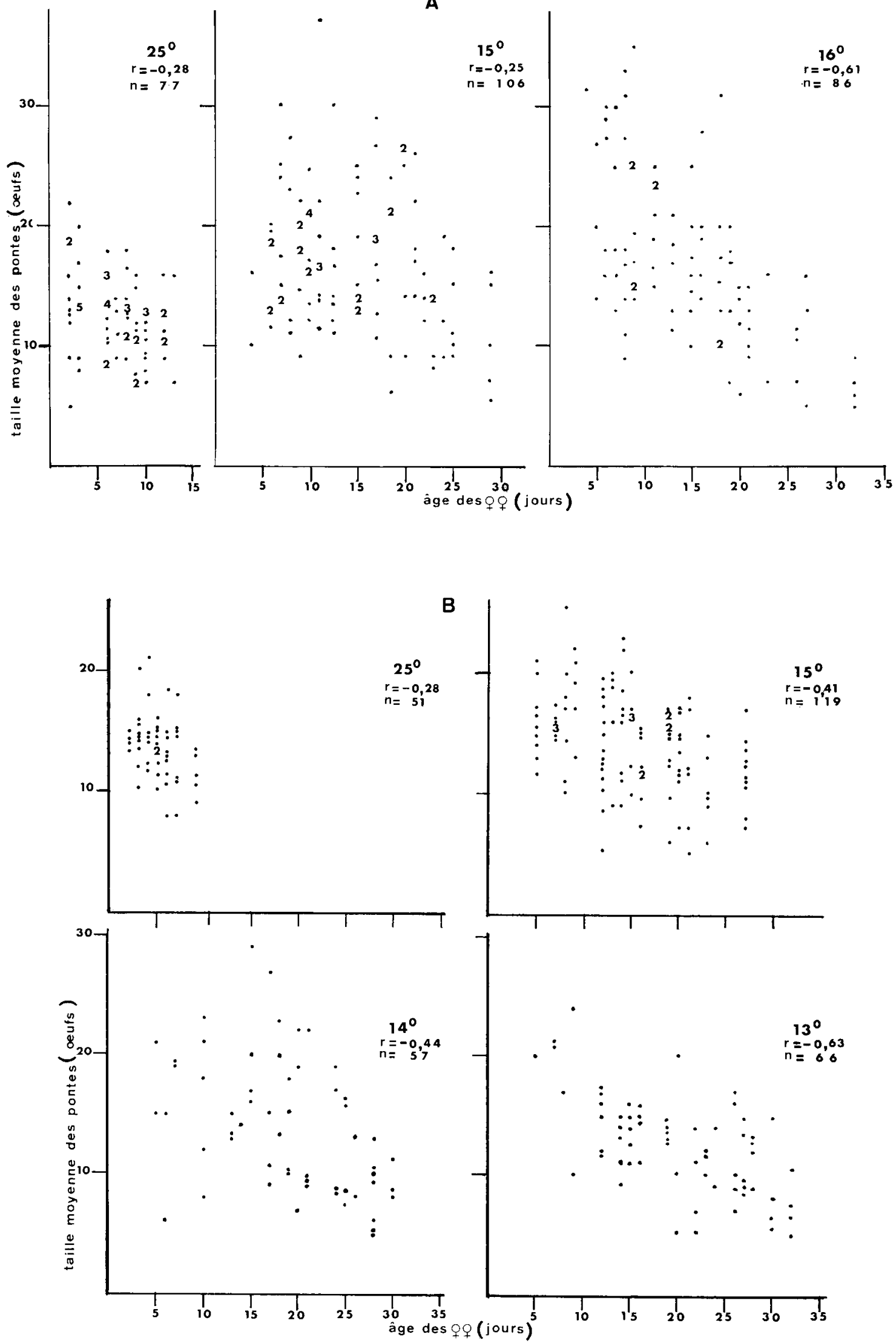

Figure 3

Relation entre le nombre moyen d'œufs par ooplaque et l'âge des femelles. Chaque point représente la taille moyenne de la ponte en terme d'œufs à un âge donné pour une femelle $\left(A: 1982 ; 25^{\circ}, 16^{\circ}\right.$, $15^{\circ} \mathrm{C} ; B: 1983 ; 25^{\circ}, 15^{\circ}, 14^{\circ}$ et $13{ }^{\circ} \mathrm{C}$ ). Lorsqu'au même âge, plusieurs femelles ont un même nombre d'oeufs par ooplaque, le nombre de ces femelles est indiqué par un chiffre. $r=$ coefficient de corrélation, $n=$ nombre total de femelles.
Relation between average number of eggs per egg-mass and female age. Each point represents one female. When more than one female had the same average egg-mass size, the number of females is indicated $(A, 1982 ; B, 1983) . r=$ correlation coefficient, $n=$ total number of females. 
port aux travaux de ces auteurs, les différents aspects de l'activité reproductrice de la pyrale du maïs abordés dans l'étude présentée ici, apportent des éléments supplémentaires sur le mode d'action de la température et des éléments nouveaux sur la ponte de la pyrale originaire du nord de la France.

Il a été ainsi constaté que l'activité de ponte subit un changement brusque de 15 à $14^{\circ} \mathrm{C}$ en 1982 et de 13 à $12^{\circ} \mathrm{C}$ en 1983 , tant sur l'intensité et le rythme, que sur la qualité des œufs. Il est évident qu'il n'y a pas eu un arrêt total de la ponte mais une modification radicale de ses composantes entre 14 et $15^{\circ} \mathrm{C}$ en 1982 et entre 12 et $13^{\circ} \mathrm{C}$ en 1983 . Malgré la différence observée au cours de ces 2 années concernant la température à laquelle a lieu une rupture dans l'intensité de l'activité de ponte, on constate un point commun relatif à la manière dont cette activité diminue en fonction de la température. Dans les 2 cas, l'activité de ponte baisse graduellement jusqu'à un certain niveau thermique et ensuite elle devient négligeable pour un écart de $1{ }^{\circ} \mathrm{C}$. La longévité étant plus grande aux températures les plus basses et l'activité de ponte $s$ 'étale sur une période plus longue.

Les grands traits qui se dégagent de cette étude en ce qui concerne les effets des plus faibles températures testées ( 13 et $14^{\circ} \mathrm{C}$ en 1982 et 11 et $12^{\circ} \mathrm{C}$ en 1983) sont :

- un nombre très restreint de femelles pondeuses,

- une réduction massive de la quantité d'œufs déposés,
- un rythme d'activité très irrégulier,

- une augmentation de la stérilité des œufs,

- une baisse du nombre d'accouplements observés à 11 et $12^{\circ} \mathrm{C}$ en 1983 .

Enfin, il faut insister sur la relativité des valeurs des températures qui ont une action notable sur la baisse de l'activité reproductrice de la pyrale, qui est évidente d'une part quand on compare les résultats de VANCE (1949) aux nôtres (les uns et les autres acquis sur des pyrales d'origine géographique différente) d'autre part quant on voit la différence observée au cours des 2 années consécutives sur la pyrale originaire de la même région du nord de la France. Il resterait à déterminer l'origine de cette différence. Etant donné que les pyrales étudiées ont passé leur vie larvaire dans la nature, on peut penser que les conditions climatiques qui ont eu lieu au cours de leur développement larvaire sont, dans une certaine mesure, à l'origine des différences de comportement des adultes observées aux basses températures.

Pour compléter cette étude, la valeur des seuils thermiques inférieurs reste à préciser puisqu'il n'y a pas eu arrêt total des caractères quantifiés aux températures testées, alors que l'arrêt de ponte était effectif à $15,4{ }^{\circ} \mathrm{C}$ pour les pyrales américaines originaires de l'Ohio, étudiées par VANCE (1949).

Reçu le 19 février 1986. Accepté le 8 août 1986.

\section{RÉFÉRENCES BIBLIOGRAPHIQUES}

Apple J. W., 1952. Corn borer development and control on canning corn in relation to temperature accumulation, J. econ. Entomol, 45, 877-879.

Barber G. W., 1925. A study of the cause of decrease in infestation of the European corn borer (Pyrausta nubilalis $\mathrm{Hbn}$ ) in the New England Area during 1923. Ecology, 6, 39-47.

Broersma D. B., Barrett J. R., Sillings J. O., 1976. Activity and black light induced flight of black cutworm and European corn borer as related to temperature and relative humidity. Environ. Entomol., 5, 1191-1194.

Caffrey D. J., Worthley L. H., 1927, in Apple J. W., 1952. Corn borer development and control on canning corn in relation to temperature accumulation. J. econ. Entomol., 45, 877-879.

Clement S. L., Rubink W. L., Rings R. W., Casey M. A., 1981. Predicting flight activities of the European corn borer. Ohio. Rep. Res. Dev., 66, 3-4.

Gahukar R. T., 1976. Contribution à l'étude de la nutrition de la pyrale, Ostrinia nubilalis Hübner (Lepidoptera : Pyraustidae) et de la résistance du maïs (Zea mays L.) à cet insecte. Thèse de Doctorat d'état, Université de Paris XI, Centre d'Orsay, $104 \mathrm{p}$.

Guennelon G., Audemard H., 1960. La pyrale du Maïs (Ostrinia nubilalis $\mathrm{Hbn}$.) (Lépidoptère, Pyralidae) dans la basse vallée du Rhône : observations écologiques, incidences économiques. Ann. Epiphyt., 11, 337-396.

Hawlitzky N., 1986. Etude de la biologie de la pyrale du maïs, Ostrinia nubilalis $\mathrm{Hbn}$. (Lep. Pyralidae), en région parisienne durant quatre années et recherches d'éléments prévisionnels du début de ponte. Acta Oecol. Oecol. appl., 7, 47-68.
Jackson R. D., Peters D. C., 1963. Biological observations on the European corn borer in southeastern Missouri. J. econ. Entomol., 56, 741-747.

Jarvis J. L., Brindley T. A., 1965. Predicting moth flight and oviposition of European corn borer by the use of temperature accumulation. J. econ. Entomol., 58, 300-302.

Loewer O. J., Huber R. T., Barrett J. R., Peart R. M., 1974. Weather-based simulation of an insect population (European corn borer). Simulation, 22, 113-118.

Matteson J. W., Decker G. C., 1965. Development of the European corn borer at controlled constant and variable temperatures. J. econ. Entomol., 58, 344-349.

Pesho G. R., 1961. Female mating patterns and spermatophore counts in the European corn borer, Pyrausta nubilalis (Hübner). Proc. North cent. Branch. Entomol. Soc. Amer., 16, 43.

Stengel M., 1982. Essai de mise au point de la prévision des dégâts pour la lutte contre la pyrale du mais (Ostrinia nubilalis) en Alsace (Est de la France). Entomophaga, 27 ( ${ }^{\circ}$ HS), 105-114.

Stirrett G. M., 1938. A field study of the flight, oviposition and establishment periods in the life cycle of the European corn borer, Pyrausta nubilalis $\mathrm{Hbn}$. and the physical factors affecting them. $V$. The seasonal characteristics of flight, oviposition and larval establishment. The variations and effects of seasonal climate. The factors causing fluctuations in borer populations. Sci. Agric., 18, 656-683.

Vance A. M., 1949. Some physiological relationships of the female European corn borer moth in controlled environments. J. econ. Entomol., 42, 474-484. 